Binghamton University

The Open Repository @ Binghamton (The ORB)

Philosophy Faculty Scholarship

Philosophy

Winter 1-1-2007

\title{
Trading Quality for Quantity
}

Christopher Morgan-Knapp

c.morgan-knapp@binghamton.edu

Follow this and additional works at: https://orb.binghamton.edu/philosophy_fac

Part of the Philosophy Commons

\section{Recommended Citation}

Journal of Philosophical Research Volume 32, 2007 Christopher Knapp Pages 211-233 https://doi.org/ $10.5840 / j p r 20073243$

This Article is brought to you for free and open access by the Philosophy at The Open Repository @ Binghamton (The ORB). It has been accepted for inclusion in Philosophy Faculty Scholarship by an authorized administrator of The Open Repository @ Binghamton (The ORB). For more information, please contact ORB@binghamton.edu. 
This is an Author's Manuscript version of the following article, accepted for publication in

Fournal of Philosophical Research, Volume 32, 2007.

https://wewre.pdonet.org/jpr/content/jpr_2007_0032_0211_0233

It is deposited under the terms of the Creative Commons Attribution-NonCommercial License (http://creativecommons.org/licenses/by-nc/4.0/), which permits non-commercial re-use, distribution, and reproduction in any medium, provided the original work is properly cited.

\title{
TRADING QUALITY FOR QUANTITY*
}

\section{CHRISTOPHER KNAPP}

\author{
Binghamton University
}

1. Some philosophical problems are decidedly esoteric. One could be forgiven for thinking that certain counterexamples Larry Temkin and Stuart Rachels have offered to the transitivity of the better-than relation are prime instances. For the most compelling of these putative counterexamples rely on our evaluative judgments concerning the quality of hypothetical lives that last an incredibly long time. And if the phenomena the counterexamples purport to uncover emerge only when we evaluate lives that last, say, $10^{50}$ years, it might seem that, for all practical purposes, they are phenomena that could safely be ignored. ${ }^{1}$

Such complacency would be a mistake. Although I will argue that these putative counterexamples need not lead us to reject the transitivity of the better-than relation, they do concern a common, and quite important, feature of our evaluative lives. More specifically, they are instances of our practice of taking certain qualitative differences between options to carry a particular kind of evaluative significance. The primary goal of this paper is to begin the work of developing an account of this practice: what it is, what difficulties it engenders, and how those difficulties might be addressed. The paper begins with an attempt to both illustrate and clarify the role qualitative distinctions play in some of our evaluative judgments. I then go on to show that our practice of according them this role runs into trouble when our options represent borderline cases of evaluatively significant qualitative distinctions. The bulk of the third section of the

* For comments on previous versions of this paper, I am grateful to Charles Goodman, an anonymous referee for this journal, and, especially, Larry Temkin.

1 The counterexamples are presented in Temkin (1996) and Rachels (1998). Rachels is sympathetic to the view that the counterexamples are not of great practical import: "Abandoning Transitivity has few practical implications. Because there are few exceptions to the principle, it may persist in our reasoning as a rule of thumb." (Rachels (1998), p. 83) 
paper is dedicated to motivating an analysis of the evaluative status of such options. There I lay the groundwork for saying that such options generate comparisons in which we can neither assert nor deny that one option is better than another. The fourth section of the paper shows how this analysis gives us hope of defusing Temkin's and Rachels' argument against the transitivity of the better-than relation. Finally, I close with a discussion of how the account developed here might go beyond resolving theoretical puzzles and be of practical import in situations that confront us in ordinary life.

2. In making practical decisions, we often must make judgments about the comparative value of our options. Rarely are these judgments based on a single kind of evaluative consideration. Instead, there are typically several different evaluative dimensions along which two options must be compared in order to determine whether one is better or that they are equally good. Take a choice that is undoubtedly familiar to many of us - that of what kind of life to lead. In order to determine whether one kind of life is better for us than another, we need to assess the degree to which each provides opportunities for meaningful relationships, accomplishment, contentment, health, and many other things that can make a life good. Or, to use a less egocentric example, take the choice of which government policies to support. In order to determine whether one policy is better than another, we need to assess the degree to which each expresses respect for those it would affect, honors the valid claims that people have, will make the world a better place, and so on. ${ }^{2}$ In these cases, and countless others, determining our options' comparative value requires not only measuring the options along each evaluative dimension, but also determining how these several measurements combine to produce an all-things-considered comparative valuation.

What often makes these latter determinations so hard is that one option will be better than an alternative in one evaluative dimension, but worse in another. In these cases, we might say, there are evaluative tradeoffs. Each option "trades" losses in one kind of value for gains in another, relative to the alternative. Returning to our examples, a life in which developing one's creative talents takes a primary role might involve a tradeoff of contentment for accomplishment when compared to a life guided by the appreciation of beauty. Or an affirmative-action policy might trade off the honoring of valid claims for an improvement in equality when compared to a policy based purely on merit. Where there are such tradeoffs, determining whether one option is better than, worse than, or equally good as an alternative thus involves a determination of whether the alternative's comparative gains in some evaluative dimensions offset its losses in others.

To complicate matters even further, there are different kinds of tradeoffs. This is so because there are both "quantitative" and "qualitative" differences in value. When options differ only quantitatively along an evaluative dimension, one option

${ }^{2}$ In both these examples, the evaluative dimensions I mention all seem to represent distinct values. This is not always the case: as we shall see shortly, we sometimes must consider the degree to which our options possess different aspects of the same value. 
simply has more of the relevant evaluative consideration than an alternative option; the difference between them is simply a matter of degree. For instance, one life may include more creativity than another; one policy may produce a more equal distribution of goods than another. When options differ qualitatively along an evaluative dimension, on the other hand, there is a difference in kind between two alternatives with respect to the relevant evaluative consideration. For instance, one life may be a happy one and another an unhappy one; one policy may be just while an alternative is unjust.

Although the point is obvious, it is helpful to keep in mind that qualitative differences in a certain value are also quantitative differences in that value. Some quantitative differences in value are sufficient to generate qualitative differences in value, while others are not. If we take the evaluative dimension of contentment as an example, we can represent this aspect of its structure graphically as:

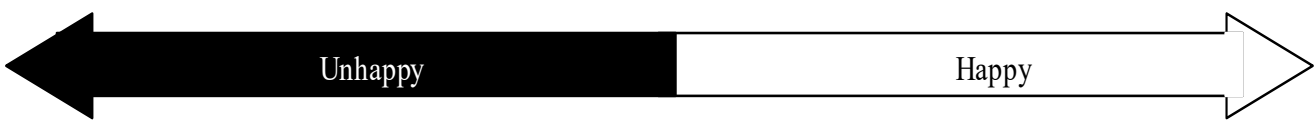

\section{Contentment}

Fig. 1

Or to put the point of the figure verbally, we say that as the amount of contentment in a life increases, eventually it possesses enough contentment to qualify as a happy life.

Our intuitions concerning whether a given tradeoff makes an option better than an alternative are often sensitive to whether or not a qualitative difference in value is at stake. Imagine, for instance, three levels of contentment that a life might possess - A, D, and $\mathrm{E}$ - whose relationships could be represented as follows (levels $\mathrm{B}$ and $\mathrm{C}$ will be considered shortly):

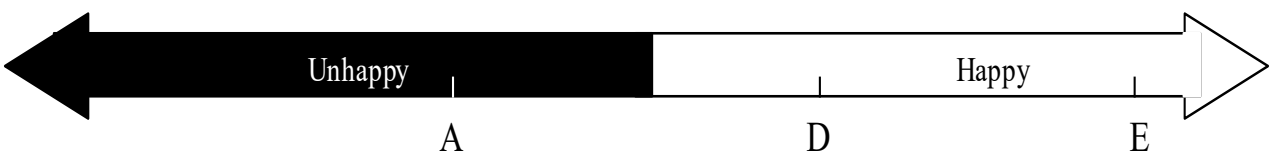

Contentment

Fig. 2

That is, a life with contentment-level $\mathrm{E}$ contains more contentment than one with level D; a life with level D contains more contentment than a life with level A; and a life with level $\mathrm{D}$ or $\mathrm{E}$ qualifies as a happy life, while a life with level $\mathrm{A}$ is an unhappy one. Now imagine two different levels of accomplishment - say, producing a body of work that has an impact on one's field in one's own time, and producing a body of work whose impact continues to be felt fifty years after one's death. If the sacrifice in contentment that would be necessary to produce the 
greater of these accomplishments meant leading a life with contentment-level D rather than level $\mathrm{E}$, many of us can imagine that that this tradeoff could make one's life better overall - depending, probably, on how much of a difference in contentment there is between these two happy lives. Producing such a long-lasting body of work, we might well think, could be worth leading a less contented, but still happy, life. If, however, producing the greater of these two accomplishments meant that one would live a life with contentment-level A rather than one with level D, many of us might judge that this tradeoff of contentment for accomplishment must not only fail to make one's life better, it must make one's life worse. For us, being unhappy detracts too much from the overall quality of a life to be made up for by an additional fifty years of posthumous influence. Let us call this kind of case one in which the presence of a qualitative difference in one evaluative dimension constitutes a barrier to improvement by some quantitative improvement in another dimension - or a "qualitative barrier case," for short. In qualitative barrier cases, then, we say that a qualitative tradeoff in one evaluative dimension for a given improvement in another could not make an option better, all things considered (indeed, it will make it worse), but a merely quantitative tradeoff for the given improvement might. ${ }^{3}$

Intuitive examples of qualitative barrier cases are not hard to come by. Indeed, we even have an idiom in English for calling attention to their existence. When faced with the prospect of having to make tradeoffs, we often report our unwillingness to make tradeoffs that are qualitatively significant by saying, "I draw the line at ..." where we fill in the ellipsis with a qualitatively significant demarcation. To cite just a few more examples: Many of us might think it is better to support a less than perfectly meritocratic policy if it produces a more equal distribution of goods than would a policy that gives everyone precisely what they deserve, but we would "draw the line" at any policy that departs so far from the demands of desert that it is unjust. Many of us might think that it is better to be less than straightforward in order to help a friend avoid a certain embarrassment, but we would "draw the line" at telling an outright lie. Many of us might think that it is sometimes better to set aside the less beautiful of two tracts of land as a park if it is more accessible to a certain number of people, but we would "draw the line" at setting aside an area so aesthetically inferior that it is ugly. And many of us might think it would be better to tackle problems that admit of a certain degree of clarity and precision at some expense of worldly relevance, but we would "draw the line" at working on problems that were, practically speaking, irrelevant.

${ }^{3}$ Here is a more formal specification of the structure of qualitative barrier cases. A choice between two options, $\mathrm{X}$ and $\mathrm{Y}$, will qualify as a qualitative barrier case if and only if there are two evaluative dimensions, $\mathrm{V}_{1}$ and $\mathrm{V}_{2}$, such that:

(a) if $\mathrm{X}$ were merely quantitatively better than $\mathrm{Y}$ with respect to $\mathrm{V}_{1}$ to a certain degree, and merely quantitatively worse than $\mathrm{Y}$ with respect to $\mathrm{V}_{2}$, then, ceteris paribus, $\mathrm{X}$ could be better than $\mathrm{Y}$, all things considered, and

(b) if $\mathrm{X}$ were merely quantitatively better than $\mathrm{Y}$ with respect to $\mathrm{V}_{1}$ to the same degree, but qualitatively worse than $\mathrm{Y}$ with respect to $\mathrm{V}_{2}$, then, ceteris paribus, $\mathrm{X}$ must be worse than $\mathrm{Y}$, all things considered. 
In our original case of trading contentment for accomplishment, there seems to be a qualitative barrier that makes it impossible for relatively pedestrian accomplishments to compensate for living an unhappy life. Nonetheless, it may be that an unhappy life that contains a truly great accomplishment is better than a happy life that lacks any significant accomplishment whatsoever. The qualitative barrier blocks a range of tradeoffs of contentment for accomplishment, but not all such tradeoffs. It seems, however, that there are cases in which the qualitative barrier is far more robust. Indeed, many believe that there are cases in which trading off a qualitative difference in one evaluative dimension for gains in another could not make an option better, no matter how much gain in the other dimension is made. As one example of such a lexical approach to some qualitative tradeoffs (and one that figures in the challenge to the transitivity of 'better than' I mentioned earlier), consider what Larry Temkin says about the relationship between the intensity of pains and their duration:

In essence, I think significant amounts of torture have lexical priority over any amounts of a hangnail.... My model for this is something like the following. Torture's badness might range from 0 to 10 , depending on its duration, with two years of torture being, say, a 7. A hangnail's badness might range from 0 to 1 . Prolonging a hangnail increases the value of the decimal places representing its "badness score," but the fundamental gap between 1 and 7 is never affected. ${ }^{4}$

According to Temkin, the qualitative tradeoff of a life marred by a mere hangnail for one that contains two years of torture could never make a life better, no matter how much longer the pain of the hangnail lasts. But, as we shall see shortly, it seems undeniable that the merely quantitative tradeoff of a life with tortuous pain for a life with a pain that is slightly more tortuous but in which the pain lasts a much shorter time could make for a better life overall. Thus, whether trading a life that includes a certain pain for one that includes a more intense but shorter pain makes one's life better depends on whether there is a particular qualitative difference in intensity between the two pains. This sort of case is different from ordinary qualitative barrier cases we have been discussing, in that there is no restriction concerning the amount of quantitative improvement in one evaluative dimension that might be gained in exchange for the qualitative sacrifice in another: trading a life with a hangnail for a life with two years of torture will always make a life worse, no matter how long the hangnail would have lasted. Let us call cases with this structure "lexical qualitative barrier cases."

The intuitions that generate lexical qualitative barrier cases will be familiar to moral theorists, for they lie behind certain popular "anti-aggregationist" - or, more precisely, anti-additive aggregationist - views. According to such views, there are pairs of kinds of values such that no amount of one kind of value can outweigh any amount of the other. For instance, this relationship seems to hold between freedom from extreme pain and mild enjoyments. To borrow an example from Scanlon:

4 Temkin (1996), p. 191. 
Suppose that Jones has suffered an accident in the transmitter room of a television station. Electrical equipment has fallen on his arm, and we cannot rescue him without turning off the transmitter for fifteen minutes. A World Cup match is in progress, watched by many people, and will not be over for an hour. Jones' injury will not get worse if we wait, but his hand has been smashed and he is receiving extremely painful electrical shocks. Should we rescue him now, or should we wait until the match is over? Does the right thing to do depend on how many people are watching - whether it is one million or five million or one hundred million? It seems to me that we should not wait, no matter how many viewers there are.... ${ }^{5}$

Here again, it seems that since the pain of severe electrical shocks is qualitatively different from the pain of missing fifteen minutes of soccer-match-viewing, there is no number of people such that it would be better to bring it about that they see the match in its entirety rather than to save a single person from such shocks. But a merely quantitative tradeoff in the intensity of pain one relieves can be justified if one is thereby able to relieve more people's pain: there is some number of people such that it would be better to save them from extremely painful electrical shocks rather than to save a single person from slightly more painful electrical shocks. Thus, the motivating intuition in this case also serves to affirm the existence of lexical qualitative barrier cases.

Despite the strength of these intuitions, the existence of lexical qualitative barrier cases is not uncontroversial. Most of the controversy arises from the fact that, in at least some cases, embracing the existence of a qualitative barrier to trading off a particular loss in one evaluative dimension for any amount of gain in another seems to commit one to rejecting the transitivity of the better-than relation. ${ }^{6}$ And while it may be plausible to say, for instance, that a several million-year-long life with a hangnail is better than a similarly lengthy life without a hangnail but with two years of torture, to some it seems even more plausible to say that if $\mathrm{A}$ is better than B and B is better than $\mathrm{C}$ then A must be better than C. However, I will argue that, properly understood, qualitative barrier cases need not threaten transitivity. If I am right, then, this central reason for doubting the existence of such barriers has no force.

3. In both the ordinary and lexical qualitative barrier cases, whether one option is better than another depends on whether or not there is a qualitative difference at stake between them. For some pairs of options, however, it is not clear that there either is or is not a particular qualitative difference at stake between them. This is

${ }^{5}$ Scanlon (2001), p. 235. Although this is a powerful case in support of anti-aggregationist views concerning the goodness of outcomes, Scanlon does not use it in this way; instead, he uses it to motivate anti-aggregationist views concerning the treatment we owe one another. For other expressions of the appeal of anti-aggregationism, see Griffin (1986), pp. 86-87; Parfit (1987), pp.160-161; and Temkin (1996), pp. 188-189.

${ }^{6}$ See, for instance, Norcross (1997), especially pp. 144-158. 
because the terms we use to mark many qualitative differences are vague. There seems to be, for instance, no precise minimum amount of contentment that one's life must contain if it is to be a happy one. There are borderline cases of happy lives, just as there are borderline cases of just policies, lying, ugliness, practical irrelevance, torturous pain, and many other markers of qualitative value differences. Again using contentment as an example, a more accurate graphical representation of the structure of these evaluative dimensions would be:

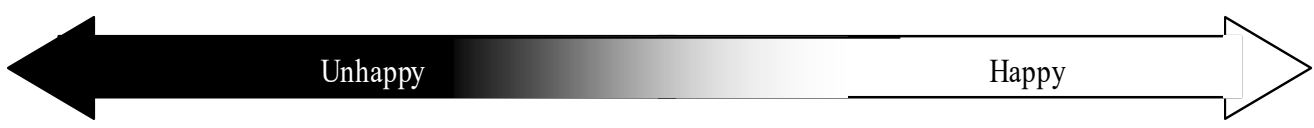

\section{Contentment}

Now, if we return to our example of trading off contentment for accomplishment, we can imagine five levels of contentment - A, B, C, D and E-that are related as follows:

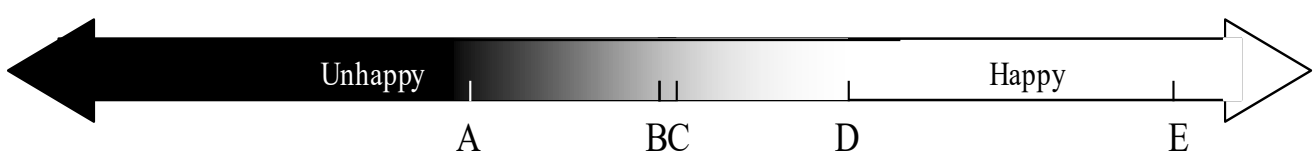

Contentment

Fig. 4

Lives with contentment-levels $\mathrm{B}$ and $\mathrm{C}$ are borderline cases of happy lives, and lives with contentment-level $\mathrm{C}$ are just ever so slightly happier than those with contentment-level B. Earlier, I claimed that trading contentment-level E for D in order to achieve fifty years of posthumous influence might make for a life that is better overall, but that trading contentment-level $\mathrm{D}$ for level $\mathrm{A}$ in exchange for the greater accomplishment would mean living a worse life. But now what do we say about trading contentment-level $\mathrm{D}$ for level $\mathrm{B}$ in exchange for the greater accomplishment? Or level B for level A? Or, for that matter, level C for level B? As we shall see shortly, answering these questions will prove important to resolving both theoretical and practical problems.

We can begin answering these questions by noting that, with respect to the tradeoffs of D for B and B for A, it seems that we can neither affirm nor deny that there is the qualitative difference between an amount of contentment sufficient for a happy life and an amount that is insufficient for a happy one that, intuitively, plays such an important role in the comparative value of two lives. This is because we can neither affirm nor deny that a life with contentment-level $\mathrm{B}$ is a happy one. The fact that one of our options is a borderline case of a qualitatively significant 
demarcation frustrates our being able to say that the relevant qualitative difference obtains between it and another. Thus, the choice between B and D, and that between $\mathrm{A}$ and $\mathrm{B}$, seem to be borderline cases of a choice in which the relevant qualitative difference is at stake. ${ }^{7}$ (That there should be such borderline cases of there being a particular qualitative difference should come as no surprise - it is hard to see how 'qualitative difference' could be precise when the terms marking the qualitative difference are vague.)

There is an intuitively appealing argument that supports this intuitively compelling view. To affirm that the relevant qualitative difference obtains between, say, contentment-level $\mathrm{D}$ and $\mathrm{B}$ would be to affirm that a life with level $\mathrm{D}$ is a happy one, while a life with $\mathrm{B}$ is not. But we cannot affirm the latter conjunct: since a life with contentment-level B is a borderline case of a happy life, we cannot claim that such a life is unhappy. Consequently, we cannot affirm that the relevant qualitative difference is at stake when one trades contentment-level D for B. Nor, for similar reasons, can we deny that the relevant qualitative difference is at stake between $\mathrm{D}$ and $\mathrm{B}$. To deny this would be to affirm that it is not the case that a life with contentment-level D is a happy life, while a life with level B is an unhappy one. And this is to affirm that either a life with level D is not a happy life or that a life with level B is not an unhappy one. But we cannot affirm either disjunct: Because a life with level D is a happy life, we cannot affirm that it is not, and because a life with level B is a borderline case of a happy life, we cannot say that is not an unhappy one. So we cannot deny that the relevant qualitative difference is at stake in the trade of $\mathrm{D}$ for $\mathrm{B}$. Thus, since we can neither affirm nor deny that the relevant qualitative difference is at stake between $\mathrm{D}$ and $\mathrm{B}$, we can conclude that trading $\mathrm{D}$ for $\mathrm{B}$ in order to gain in some other evaluative dimension is a borderline case of a tradeoff in which the relevant qualitative difference is at stake. $^{8}$

${ }^{7}$ I use the term 'the relevant qualitative difference' to refer to the difference that generates a qualitative barrier in the case under discussion. In the present context, therefore, it refers to the difference between a happy and an unhappy life.

8 This is not to say that there is no qualitative difference between contentment-levels D and B - perhaps borderline happy lives and definitely happy lives differ in the kind, not just the degree, of contentment they possess. If so, there is the further question of whether this difference generates a barrier to trading off contentment for accomplishment. If this difference does not generate such a barrier, then this qualitative difference is not evaluatively significant - trading a happy life for a borderline happy life can be treated just like tradeoffs that differ only in the degree of contentment they contain. If, on the other hand, the difference between lives that are definitely happy and those that are borderline happy does generate a barrier to trading off contentment for accomplishment, then precisely the same points under discussion here will arise again: this time for borderline cases of borderline happy lives. For the division between borderline happy lives and definitely happy lives is no sharper than that between happy lives and unhappy ones: 'borderline happy' is, like 'happy,' a vague term. For more on 
While I do ultimately find this argument persuasive, not all arguments of this form will be persuasive as well. For the present argument infers from the fact that we can neither affirm nor deny two disjuncts that we can neither affirm nor deny their disjunction. But this is not universally the case. It may be, for instance, that I can neither affirm nor deny that the Riemann Hypothesis is true, and that I can neither affirm nor deny that it is false. Nonetheless, I can affirm that the theorem is either true or false.

There is, however, an important difference between the argument I offered above and the mathematical counterexample to the general argument form. In the mathematical counterexample, we know that the Riemann Hypothesis is not a borderline case of a true theorem, for there are no borderline cases of true theorems. And it is my confidence that this is so that explains why I can affirm that the theorem is either true or not, despite my not being able to say which. But the argument concerning whether the relevant qualitative difference is at stake between contentment-levels $\mathrm{D}$ and $\mathrm{B}$ cannot be undermined by similar reasoning. It is precisely because the disjunction 'Either a life with contentment-level D is not a happy life or a life with level B is not an unhappy one,' contains a disjunct that is a predication of a term to one of its borderline cases that we are led to believe that we can neither affirm nor deny the disjunction.

Part of the difficulty here is that my characterization of borderline cases of a vague term as cases in which we can neither affirm nor deny that the term applies is neutral between several different explanations of why this might be so. This neutrality has been helpful because, in identifying a central aspect of borderline cases that is nearly universally granted by philosophical accounts of vagueness, it allows our discussion to proceed without taking a stand on controversial matters in semantics. But because the precise structure of a successful argument for the claim being defended here - viz. that a tradeoff involving a borderline case of an evaluatively significant qualitative distinction is a borderline case of a tradeoff in which that qualitative difference is at stake - will depend on how we understand borderline cases, this neutrality prevents the intuitive version of the argument from being as clean as one might like. ${ }^{9}$ So in order to allay any further doubts, let us go through the three main contenders for the semantics of vague predicates supervaluationism, epistemicism and many-valued logics - to assure ourselves that each of their ways of clarifying the intuitive argument preserves its force.

Let us start with supervaluationism. According to this family of theories, we can neither affirm nor deny that a term applies to one of its borderline cases because it is neither true nor false that it applies. This is because our linguistic practices fail to precisely delimit a vague term's extension. They do, however, determine a

this phenomenon of higher-order vagueness, see the discussions in Williamson (1994) and (1999).

${ }^{9}$ Here, and throughout, I use "evaluatively significant qualitative distinction" to refer to qualitative distinctions that generate qualitative barrier cases. 
range of "admissible" extensions - precise extensions that are consistent with how the term is ordinarily used. Sentences containing vague terms are true just in case they are true according to each of the admissible extensions, false just in case they are false on all admissible extensions, and indeterminate otherwise. Borderline cases of a term are cases that are contained in some, but not all admissible extensions of the term. Consequently, the predication of a vague term to one of its borderline cases will be true according to some, but not all of the admissible extensions. Such predications are, therefore, indeterminate. ${ }^{10}$ Given such an account, it is not hard to justify the intuitive argument in question. As that argument suggests, we can neither affirm nor deny that a particular qualitative difference obtains between a clear case of the relevant qualitative term and a borderline case of that term. This is because the claim that the difference obtains is true on some admissible extensions of the term - namely, those that do not include the borderline case - and false on others - namely, those that do include the borderline case - and hence is itself indeterminate. Thus, for supervaluationists, tradeoffs involving a borderline case of a qualitative distinction - such as the tradeoff of contentment-level D for contentment-level B - are borderline cases of tradeoffs in which that qualitative difference is at stake.

Epistemicists, on the other hand, deny that any sentence is indeterminate in the supervaluationist's sense. This is because epistemicists hold that vague terms do have precisely specifiable extensions; it is just that we cannot precisely specify them. ${ }^{11}$ Borderline cases of vague predicates are cases in which we are irredeemably ignorant of whether the predicate applies rather than cases in which there is no fact of the matter about whether the predicate applies. There is, for instance, a precise minimum amount of contentment that is necessary for leading a happy life, but, unfortunately, determining what this minimum amount is lies beyond our ken. Contentment-level B could be at or above this minimum amount or not. If it is below the minimum, then it is true that trading $\mathrm{D}$ for $\mathrm{B}$ is to trade a happy life for an unhappy one and thus to make a trade in which there is qualitative difference at stake; and if B is at or above the minimum amount, it will be false that the trade is of this sort. We are not, however, in a position to know either way, and, consequently, we are likewise ignorant of whether the relevant qualitative difference is at stake between B and D. And insofar as this kind of ignorance is exactly the kind of ignorance that is characteristic of borderline cases of a predicate, the difference between $\mathrm{D}$ and $\mathrm{B}$ is, for epistemicists, a borderline case of the relevant qualitative difference obtaining.

And finally, those who support many-valued logics will also agree that the difference between $\mathrm{D}$ and $\mathrm{B}$ is a borderline instance of the relevant qualitative difference being at stake, in yet a third sense of 'borderline case.' Predications of a vague term to borderline cases, according to these theorists, are neither completely true nor completely false; they are true to a certain degree. Typically, this is represented by assigning some numerical truth-value to borderline predications between zero (which is assigned to claims that are completely false)

10 See, for instance, Fine (1975).

11 See, for instance, Williamson (1994), Chapters 7 and 8. 
and one (which is assigned to claims that are completely true). These theorists then go on to specify how the degree to which a logically complex proposition is true varies as a function of the degree to which its components are true. For instance, if we represent the degree to which an atomic sentence 'p' is true by the symbol $|p|$, standard treatments of "Not-p," "p and q," and "p or q" in manyvalued logics are:

$$
\begin{array}{ll}
\sim \mathrm{p}=1-|\mathrm{p}| & \\
\mathrm{p} \wedge \mathrm{q}= & \min \{|\mathrm{p}|,|\mathrm{q}|\} \\
\mathrm{p} \vee \mathrm{q}= & \max \{|\mathrm{p}|,|\mathrm{q}|\}^{12}
\end{array}
$$

Using a typical many-valued logic, then, one would state the intuitive argument more precisely as follows: To affirm that the relevant qualitative difference is at stake between $\mathrm{B}$ and $\mathrm{D}$ is to affirm that $\mathrm{D}$ is a happy life and $\mathrm{B}$ is an unhappy one. This conjunction, however, is only partially true. Since a life with level B is a borderline case of being a happy life, the claim that a life with level B is unhappy is only partially true, and a conjunction is only as true as its weakest conjunct. Similarly reasoning applies to the denial of there being the relevant qualitative difference between $\mathrm{B}$ and $\mathrm{D}$. To deny this is to affirm that either a life with level $\mathrm{D}$ is not a happy life or that a life with level B is not an unhappy one. But this disjunction is only partially true, for a disjunction can only be as true as its truest disjunct, and in this case the truest disjunct - namely, B is not an unhappy life - is only partially true. So the difference between $\mathrm{B}$ and $\mathrm{D}$ is a borderline case of the relevant qualitative difference.

As I mentioned before, the conclusion of our original argument, and of all these precisifications of it, is an intuitively compelling one. What makes it interesting, though, is that one can argue on the same grounds that, because lives with contentment-levels $\mathrm{C}$ and $\mathrm{B}$ are borderlines cases of being happy ones, the choice between $\mathrm{C}$ and $\mathrm{B}$ is a borderline case of a choice in which the relevant qualitative difference is at stake. This is because the argument shows that one of our option's being a borderline case of a qualitative distinction is enough to guarantee that any tradeoff involving that option will be a borderline case of a tradeoff in which that difference is at stake. And this sufficient condition is clearly satisfied in the tradeoff between B and C. (Rather than go through the argument and each precisification again, I will leave it to any doubtful readers to satisfy themselves by substituting contentment-level C for contentment-level D throughout.)

To be fair, there is an intuition that might make accepting this conclusion less than comfortable. Some will be troubled by the fact that there is only a slight difference in the degree of contentment between $\mathrm{B}$ and $\mathrm{C}$. This can be troubling insofar as we might find it implausible that a slight difference in the degree of contentment that two lives possess could possibly constitute the difference between a happy life and an unhappy one. For those with this intuition, it will seem that it must be false to say that the relevant qualitative difference is at stake when one trades contentment-level $\mathrm{C}$ for level $\mathrm{B}$. And if it is false that this qualitative

12 See, for instance, Machina (1976). 
difference is at stake, then this cannot be a borderline instance of that difference being at stake.

The reliability of this intuition should not be taken for granted, however. For we may be mistaking its not being the case that we can correctly affirm a claim for its being the case that we can correctly deny it. That is, we may be confident that, given their similarity, it would be a mistake to say that trading contentment-level $\mathrm{C}$ for level B is to trade a happy life for an unhappy one, and then go on to suppose that this warrants our being confident that it does not involve this qualitatively significant sacrifice. But this is to ignore the possibility that it may be a mistake to say both that this qualitative difference is at stake and that it is not; it is to ignore that trading $\mathrm{C}$ for $\mathrm{B}$ could be a borderline instance of this qualitative difference being at stake. That many of us overlook this possibility is not hard to imagine, for, in my experience, the notion of borderline cases of there being a qualitative difference does not play a significant role in ordinary, pre-theoretic thought and practice. Consequently, it may be that our intuition that that there is not a qualitative difference at stake between $\mathrm{C}$ and $\mathrm{B}$ is a sign, not that many of us have rejected the idea that the case is a borderline one, but that many of us have simply neglected to consider this possibility. ${ }^{13}$

It is also worth noticing that all those philosophers of language who endorse the accounts of vagueness we just discussed are willing to contradict the intuition in question. Presumably, this is because they think that paying whatever cost is involved in rejecting this intuition is well worth the benefits that their general theories provide - not least of which is the promise of being able to explain how our predication of vague terms to things to which they determinately apply is coherent. Insofar as philosophers of value also have an interest in such predications being coherent, this benefit is a reason for them to put up with the intuitive discomfort that may arise from denying the intuition as well. But in the following section, we will see that philosophers of value have an additional motivation for holding that tradeoffs between borderline cases of evaluatively significant qualitative distinctions are borderline cases of that qualitative difference being at stake - namely, that endorsing this thesis is the best way to vindicate the theoretically fundamental assumption that the 'better than' relation is transitive.

4. Our motivation for paying such close attention to the issue of whether there is a qualitative difference between borderline cases was that this issue plays in an important role in our comparative evaluations. In both ordinary and lexical qualitative barrier cases, whether trading a loss in one evaluative dimension for a

13 This error theory can be enhanced once we have endorsed a particular theory of vagueness. Supervaluationists will say that we are mistaking there being no particular instance of a qualitative difference between borderline cases with its not being the case that there exists some such instance. Epistemicists will say that we are mistaking our irremediable ignorance with respect to where the threshold is for there being no such threshold. And degree theorists will say we are mistaking less-than-full truth for falsity. 
quantitative gain in another could yield a better option depends on whether the loss involves the relevant qualitative difference. If so, then making the tradeoff in order to realize the quantitative gain in another dimension must yield a worse result; if the tradeoff involves a merely quantitative loss, on the other hand, making the trade could make things better. What we have learned so far is that these two possibilities - that the relevant qualitative difference either is or is not at stake - are not exhaustive. It could be that a tradeoff in one evaluative dimension for a quantitative gain in another is a borderline instance of there being an evaluatively significant qualitative difference at stake. What we need to see now is what implications this holds for our comparative evaluations in qualitative barrier cases.

The most straightforward thing to say, and I believe the best thing to say, is that when a tradeoff in a qualitative barrier case is a borderline instance of the relevant qualitative difference being at stake, it will also be a borderline instance of tradeoff that yields a better result. For example, if we can neither affirm nor deny that the choice between contentment-levels $\mathrm{C}$ and $\mathrm{B}$ is a choice between a happy life and an unhappy one, then we should agree that we can neither affirm nor deny that trading off $\mathrm{C}$ for $\mathrm{B}$ in exchange for 50 years of posthumous influence makes for a better life. And again, if we can neither affirm nor deny that a further departure from the demands of desert will involve trading a just policy for an unjust one, then we should agree that we can neither affirm nor deny that making this further departure in exchange for a more equal distribution of goods makes for a better policy.

Besides being straightforward, this way of understanding such tradeoffs offers us a way out of an otherwise troubling argument concerning qualitative barrier cases. The argument has been advanced by both Larry Temkin and Stuart Rachels, and begins by making three plausible claims:

(1) For any unpleasant or "negative" experience, no matter what the intensity or duration of that experience, it would be better to have that experience than to have one that was only a little less intense but twice as long.

(2) There is a continuum of unpleasant or "negative" experiences ranging in intensity, for example, from extreme forms of torture to the mild discomfort of a hangnail.

(3) A mild discomfort for the duration of one's life would be preferable to two years of excruciating torture, no matter the length of one's life. ${ }^{14}$

The trouble with these claims is that, together, they imply that the 'better than' relation is intransitive - that is, they imply that when one option, A, is better than another option, B, and B is better than $\mathrm{C}$, it need not be true that $\mathrm{A}$ is better than C. In order to see how the denial of transitivity follows from (1)-(3), consider the following way of representing claims (1) and (2):

14 Temkin (1996), p. 179. 


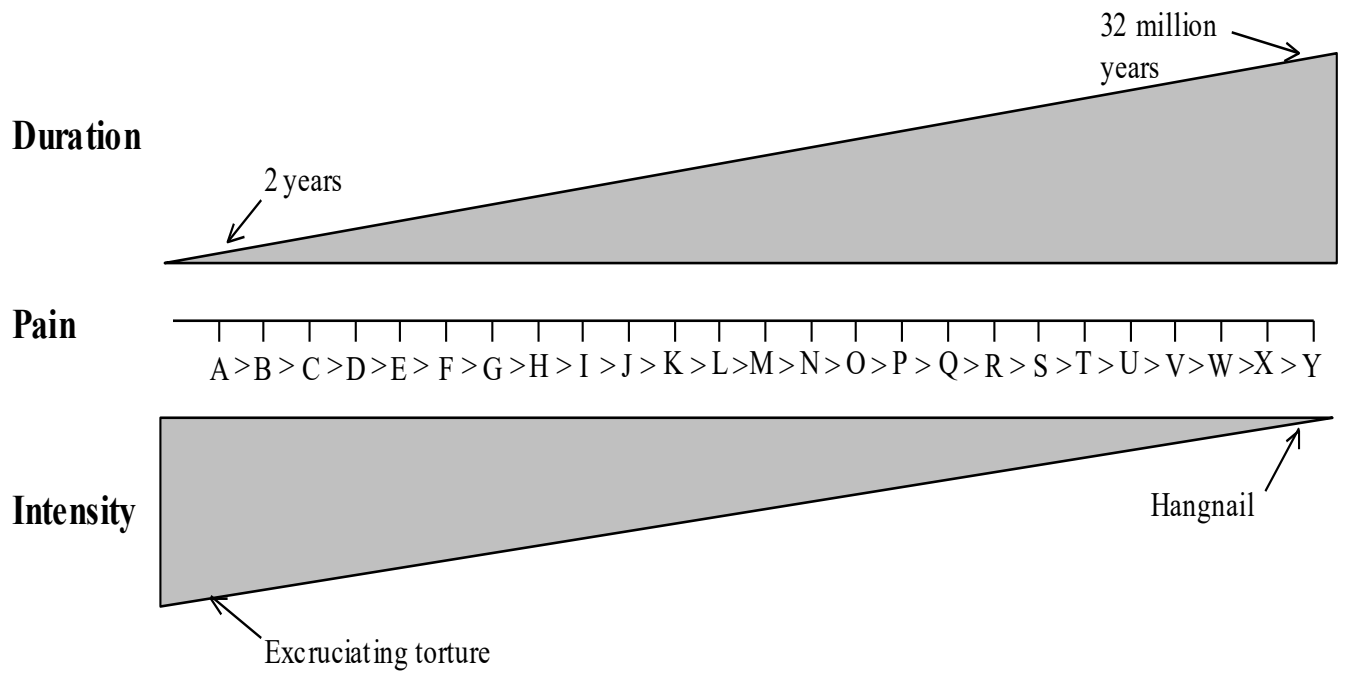

Fig. 5

Claim (2) insures that the slope of the line representing the range of pain intensity from excruciating torture down to a hangnail is smooth. And, if true, claim (1) would insure that for each pair of adjacent pains in the series from A to $\mathrm{Y}$, the pain that is slightly more intense but half as long is the better pain to experience. ${ }^{15}$ For example, claim (1) tells us that pain A (i.e., two years of excruciating torture) would be a better pain to experience than pain B (i.e., four years of slightly less excruciating torture); and it tells us that pain $\mathrm{B}$ would be better to experience than pain $\mathrm{C}$ (i.e. 8 years of torture that is slightly less excruciating than pain B); and so on down the series of pains until we reach pain Y. Now, if the 'better than' relation is transitive, this implies that pain $\mathrm{A}$ is a better pain to experience than pain $\mathrm{Y}$. But pain $\mathrm{Y}$ is the pain of a hangnail that is experienced for all of a 32 million-year long life, and claim (3) tells us that 2 years of excruciating torture must be worse than any such hangnail pain. Thus, in order to maintain claims (1)(3), we must deny the transitivity of the 'better than' relation.

To his credit, Temkin does much of the work of directing us to this argument's vulnerabilities. To begin, he points out that the argument relies on the fact that the pain of (a significant amount of) torture and a hangnail are qualitatively different, while any two adjacent pains in the series from $\mathrm{A}$ to $\mathrm{Y}$ are only quantitatively different:

Many people worry that my counterexample [to transitivity] trades on the fact that the pain of intense torture seems to be of an entirely different kind than the pain of a hangnail. This observation is, I think, right on target. ... The continuum

15 Temkin (1996) argues that dividing the continuum of pain between extreme torture and a hangnail into the 25 steps represented by options A through Y makes the steps small enough to preserve the truth of claim (1). Rachels (1998), however, suggests that with this number of steps, the difference in duration for each pair of options from A to Y might have to be 100-fold in order for the more intense but shorter pain to be better, rather 2-fold, as Temkin's claim (1) holds. 
from intense torture to a hangnail exemplifies the fact that together, a sufficient number of differences in degree can sometimes amount to a difference in kind. ${ }^{16}$

Moreover, Temkin suggests that the evaluative judgments of claims (1) and (3) are best explained by appealing to the fact that a qualitative difference is at stake in the tradeoff of torture for a hangnail, but only quantitative differences are at stake in the tradeoffs involved in each adjacent pair of pain in the series from A to Y:

In comparing pains that merely differ in degree, duration plays a significant role. That is why we think a shorter intense pain might be better than a much longer less intense pain. But in comparing pains that differ in kind, duration plays a very different role. In comparison with torture of a significant duration, a hangnail's duration basically doesn't matter. ${ }^{17}$

To put the point in our terms, trading off a less intense pain for a more intense pain in order to have a pain that lasts less long can make for a better pain overall if the tradeoff in intensity is only quantitatively significant; but if the tradeoff involves the qualitative difference between torture and a hangnail, trading off intensity for duration could not yield a better pain. That is, the argument describes a (lexical) qualitative barrier case.

And, finally, Temkin is clear that, as the intensity of pains decreases, there is no precise point at which the intensity stops being sufficient for qualifying as the same kind of pain as torture or begins being sufficient to qualify as the same kind of pain as a hangnail. If there were, the argument against intransitivity would not go through. For if the qualitative boundary (or boundaries) were precise, then there would be a pair of adjacent pains in the series from A to $\mathrm{Y}$ of which it would be true that there was the relevant qualitative difference between them, and hence false that the more intense pain would have to be better than the less intense, but longer pain. ${ }^{18}$ Putting all this together, a more accurate representation of the structure of Temkin's example is:

16 Temkin (1996), p. 194. Rachels seconds this characterization of the counterexamples, claiming that their appeal rests on principles according to which "some morally relevant differences in degree amount to morally relevant differences in kind." Rachels (1998), p. 71.

17 Temkin (1996), p. 194.

${ }^{18}$ In response to the objection that if $\mathrm{A}$ and $\mathrm{Y}$ are different kinds of pain, then there must be some point along the continuum from $\mathrm{A}$ to $\mathrm{Y}$ at which the pain ceases to be the same kind as A, Temkin writes, "The objection assumes that if two extreme ends of a continuum differ in kind, then there must be some point along the continuum whose adjacent members also differ in kind. This assumption is fallacious. From the fact that together a large number of small differences in degree can amount to a difference in kind, it does not follow that there must be some point where a small difference in degree itself results in a difference in kind. Suppose that ... there is good reason to treat a 10 -year-old sapling ... as a different kind of plant than a month-old seedling. Does that mean that there must be some point, say, between 36 and 37 months, where the seedling becomes the sapling? ... Surely not.” Temkin (1996), p. 196. 


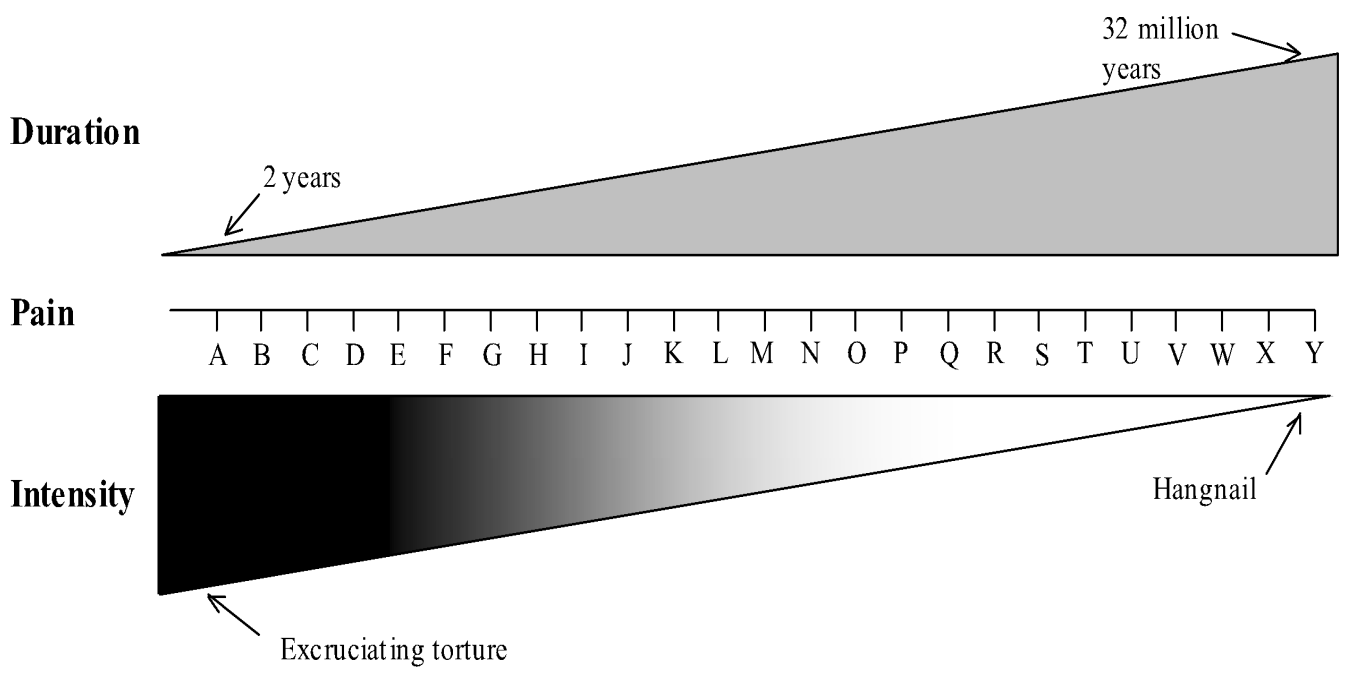

Fig. 6

By now, it should be clear how our earlier results bear on the argument for intransitivity. Since the argument appeals to a qualitative barrier case, we know that whether trading off intensity for duration yields a better pain depends on whether an evaluatively significant qualitative difference in intensity is at stake. We cannot, however, draw a precise boundary between pains that are of the same kind as excruciating torture and those that are not: There will be pains that are borderline cases of this kind of pain. In figure 6 above, pains L and M are two such cases. The argument of $\$ 2$ established that a pair of borderline cases of an evaluatively significant kind also constitutes a borderline case of the relevant qualitative difference being at stake. So we can neither affirm nor deny that the relevant qualitative difference in intensity is at stake between $\mathrm{L}$ and $\mathrm{M}-$ or, for that matter, between any adjacent pair of pains in the series from $\mathrm{A}$ to $\mathrm{Y}$ that are borderline cases of the evaluatively significant kind of pain that includes intense torture but not a hangnail. And, if we follow the suggestion at the beginning of this section, this would imply that the tradeoff of $\mathrm{L}$ for $\mathrm{M}$ would be a borderline case of a tradeoff that yields a better result. That is, we can neither affirm nor deny that $\mathrm{L}$ is better than $\mathrm{M}$. But if we can neither affirm nor deny that $\mathrm{L}$ is better than $\mathrm{M}$, then the transitivity of 'better than' will not imply that A is better than $\mathrm{Y}$, for this implication only follows if the more intense but shorter pain is positively better in each adjacent pair in the series from A to $\mathrm{Y}$.

We thus avoid being compelled to deny transitivity by recognizing that it would be a mistake to affirm claim (1). ${ }^{19}$ That is, we cannot say that is always better to

19 One might worry that our refusal to affirm or deny claim (1) will do little to undermine the force of the Temkin's and Rachels' challenge if claim (1) is nevertheless true. But this worry can be set aside, at least provided that any of the three most influential accounts of vagueness are on the right track. For according to each, (1) is not true. According to supervaluationists, (1) is false (although each of its substitution instances is neither true nor false); according to many-valued logics, (1) will be only partially true, and hence will not strictly entail the denial of 
have a slightly more intense pain if it only lasts half as long. There are, instead, some borderline cases in which we can neither affirm nor deny that this is so.

I should say that, as it stands, this is not a decisive objection to Temkin's and Rachels' argument against transitivity. This is because it assumes that the correct explanation of Claim 3 is that torture and mild discomfort are different kinds of pain. In his comments on a previous version of this paper, however, Temkin suggested a diagnosis of Claim 3 that is different than the one implied by the quotations above. According to this diagnosis, it is not the fact that torture and hangnails involve different kinds of pain that blocks the tradeoff of greater intensity for lesser duration, but that the pains differ so much. It is the size of the difference that is evaluatively significant, according to this interpretation, not the difference in kind of pain that differences of great size can generate. ${ }^{20}$

If this is the right diagnosis of our judgment, then the preceding response to Temkin's and Rachels' arguments does not undermine them. For that response trades on the fact that in the series of comparisons the argument asks us to make, we encounter borderline cases of the feature that generates the barrier to trading off lesser intensity for lesser duration - namely, of the relevant kind of pain that includes torture. If this new diagnosis is the correct one, however, Temkin's and Rachels' arguments never appeal to comparisons involving borderline cases. For on this interpretation, the relevant feature is the magnitude of the difference in intensity, and all the comparisons involve differences in intensity that are definitely minor or definitely great.

The question, then, is which diagnosis of our judgment that $\mathrm{Y}$ is better than $\mathrm{A}$ is correct. This is not an easy question to answer, since it involves disentangling two things that are present in the comparison of $\mathrm{A}$ and $\mathrm{Y}$ - both of which have an intuitive claim to evaluative significance - and determining which is responsible for making the duration of minor discomfort comparatively irrelevant. In order to directly undermine the magnitude-of-difference interpretation, we would need to see that other differences in pain intensity of roughly the same size fail to generate a barrier to trading off lesser intensity for lesser duration. But it is difficult to find such a case because it is hard to conceive of any other difference in pain intensity that is of the same size as the difference in intensity between torture and a hangnail. Alternatively, we could argue in favor of the difference-in-kind-of-pain interpretation by specifying the relevant difference, and showing that the size of the difference that is large enough to span this qualitative distinction does not generate a barrier to trading off greater intensity for lesser duration when it occurs

(2) or (3) when combined with transitivity of 'better than; and according to epistemicists, (1) is false.

${ }^{20}$ While this diagnosis of Temkin's and Rachel's qualitative barrier cases is not implausible, the corresponding diagnoses of many of our other examples of qualitative barrier cases is. In trading contentment for accomplishment, for instance, we have a special concern about losses in contentment that make us unhappy that we do not have about losses of a similar size that leave us with a happy life. 
at other points along the continuum. That is, we could specify an evaluatively significant difference in kind of pain that arises, say, in the interval between $\mathrm{F}$ and $\mathrm{O}$ in Figure 6, and then show that a similarly sized difference, say, between $\mathrm{P}$ and $\mathrm{Y}$, does not generate a barrier to trading off lesser intensity for lesser duration.

Unfortunately, I cannot yet provide such an argument, and so we are left with a choice between two competing diagnoses of Claim 3. Still, we have one powerful reason to favor the difference-in-kind-of pain explanation: it is the explanation that allows us to preserve our commitment to the transitivity of the better-than relation. Most of us are far more confident that the better-than relation is transitive than we are that the correct of explanation of Claim 3 appeals to the magnitude of the intensity difference between torture and hangnail pain rather than the fact that they are different kinds of pain. Moreover, the transitivity of the better-than relation is a fundamental axiom of decision theory and economics; no diagnosis of claim Claim 3 plays any such theoretical role. ${ }^{21}$ Thus, we have both strong intuitive and theoretical reasons to believe that the reason we prefer mild discomforts of any length to two years of torture is that discomforts and torture constitute different kinds of pain.

5. It may be tempting to imagine that the structural aspects of qualitative barrier cases we have discussed so far are of primarily esoteric interest. ${ }^{22}$ After all, while facing tradeoffs involving borderline cases of evaluatively significant qualitative distinctions may not be a rarity, our analysis suggests that whatever choice one makes in such cases, it is unlikely that one will ever go wrong. Since we can neither affirm nor deny that making these tradeoffs yields a better result, it seems that, typically, whether we make them or not is of little consequence. And so even if we erroneously believe that there is no qualitative difference at stake in such tradeoffs, and consequently believe that we ought to make them, we will be none the worse for our confusion. Of course, we should admit that it is not impossible to suffer from this confusion; if, for instance, one is faced with a sequence of tradeoffs that is ordered in the way that the options in Temkin's and Rachels' arguments are, one will wind up in trouble if one continues to tradeoff a value that generates a qualitative barrier. But how likely is it that people ever actually face such a sequence?

It is far more likely than one might have expected. In at least two common contexts, we confront sequences of tradeoffs that approximate the Temkin/Rachels series. ${ }^{23}$ And in such cases, we often make things worse for ourselves because we fail to appreciate that we can neither affirm nor deny that

${ }^{21}$ Temkin himself may not find this argument very compelling, for he believes there are independent grounds for denying transitivity. See Temkin (1987). See also Kamm (1996), Chapter 12.

${ }^{22}$ Joseph Raz expresses such an appraisal when he writes, "I am not aware of any very significant implications [the indeterminacy of value] has for practical thought." Raz (1986), p. 324.

${ }^{23}$ In recent work, Temkin has also discussed the practical significance of his continuum argument. See, for instance, Temkin (2005), §3-5. 
making tradeoffs involving borderline cases of evaluatively significant qualitative distinctions yields a better result.

One common context in which we come up against a problematic sequence of possible tradeoffs is when we must decide whether to contribute to a certain kind of collective good. Collective goods - that is, goods that, if they are available to one person in a collective, are available to all in the collective - come in several varieties. Sometimes any contribution to the production of a collective good, no matter how small, increases the amount of the good produced. For other collective goods, however, it is only after the total contributions have reached a certain magnitude that the good will be produced at all. For some of these "step" collective goods, the amount of contributions required to produce the good is precise. An example of a precise step good is the passage of a bill in a legislative assembly - there is a precise number of votes the bill must receive in order to pass. For other step goods, however, the amount of contributions necessary to produce the good is vague. An example here is the good of a healthy drinking water supply - a water supply must be largely, but not completely, free of toxins in order to be healthy, but there is no precise minimum level of toxicity at which it stops being unhealthy and begins to qualify as healthy. It is with respect to this latter, quite common, sub-category of step goods that we ignore the structure of qualitative barrier cases at our peril.

As has been widely discussed, the cooperative production of collective goods can be difficult for groups to achieve if each member of the group is free to refrain from contributing to the good. Even if all members would be better off if they all contributed to the public good than if none of them contributed, a group may still fail to produce the good because each individual will hope to profit from the contributions of others without paying the cost of contributing herself. If each individual acts on this hope of "free-riding" on the contributions of others, none will contribute, and the good will never be produced.

Notice that we can interpret the question of whether to contribute to the production of a collective step good as a question of whether to make a tradeoff in a qualitative barrier case. In qualitative barrier cases, we say that whether one should trade losses in one evaluative dimension for gains in another depends on whether there is an evaluatively significant qualitative difference at stake. In collective step good contribution decisions, we can similarly say that whether an individual should pay the cost of contributing to the collective good depends on whether there is an evaluatively significant qualitative difference at stake - namely the difference between the good's being produced and its not being produced. If an individual's contribution is not necessary for the good to be produced, the relevant qualitative difference is not at stake, and she will probably be better off saving her contribution for herself. But if her contribution is necessary to produce the good, she will be better off contributing, because failing to contribute will involve paying the qualitatively significant sacrifice of being unable to enjoy the collective good at all. 
The danger is that for vague step goods, individuals often reason as follows. Whether I should contribute depends on whether the qualitative difference between the good's being produced and not being produced is at stake. Since I cannot (rationally) produce the good myself, whether this is so depends on how many others contribute. But when I consider each possible number of other contributors, it is never the case that adding my single contribution makes the difference between the good's being produced and not. For there is no precise minimum number of contributions necessary in order to produce the good. So, since there is never an evaluatively significant qualitative difference at stake in my decision of whether to contribute, I am always better off not contributing. Not contributing is, in the game theorist's terms, my dominant strategy. ${ }^{24}$

Were we unable to object to this line of reasoning, it would be very difficult to show that individuals ever had self-interested reason to contribute to the production of vague step goods, despite the fact that, as a group, they would all be better off if they all made a contribution. And indeed, it may well be the seductiveness of such reasoning that explains why many such collective goods fail to be generated voluntarily. Fortunately, however, the present analysis allows us to make the following objection. Since the collective good at issue is vague, there will be levels of its production that count as borderline cases of the good's being produced. When, because of the contributions of others, the production of the good is at a borderline level, the decision of whether to contribute will not be a decision in which we can deny that there is an evaluatively significant qualitative difference at stake, but rather a decision in which we can neither affirm nor deny that this is so. Consequently, we can neither affirm nor deny that an individual will be better off if they contribute to the good in such situations. And so it is simply not the case that the individual will always do better by not contributing to the production of the collective good no matter how many others contribute. ${ }^{25}$

To be sure, this does not show that individuals ever have self-interested reason to contribute to the cooperative production of vague step goods. This sort of result must await an account of the reasons that exist with respect to decisions of which we can neither affirm nor deny that they would yield a better result. It is possible that an account could be developed according to which a self-interested reason to contribute arises from the fact that, from the individual's point of view, it is better if everyone, including the individual, contributes than if none do. But even if this is not so, it seems unlikely that an account of the reasons that exist in these borderline cases will have it that the individual has self-interested reason not to contribute. And in the absence of such reason, it may be easier to make individuals responsive to non-self-interested reasons - such as those generated by

${ }^{24}$ Pettit (1986) endorses the cogency of this line of reasoning. Theorists who have suspected it of being fallacious include Hampton (1987) and Tuck (1987).

${ }^{25}$ Or to put the point in game-theoretic terms, non-contribution is not the dominant strategy for each individual. Thus, vague collective step goods cannot generate bona fide prisoner's dilemmas. 
considerations of fairness or the common good - that speak in favor of contributing.

This way of understanding how we may go wrong in certain free-rider cases, and how the present account provides hope of setting us aright, can also serve as a model for our treatment of a second practical context in which we stand to gain from a better appreciation of the structure of qualitative barrier cases. In the sort of situation I have in mind, we are concerned not with a vague step good that can only be produced by the cumulative contributions of a collective, but with a vague step good that can only be produced by the cumulative contributions of a single agent over time. A good example of such a good is the good of an individual's being physically healthy. This is a vague step good because there is an evaluatively significant qualitative difference between being healthy and not being healthy that emerges over a vague interval rather than at a precise level of proper physical functioning. And it is a good that can only be achieved by repeated contributions by a single agent because, in order to be healthy, the individual must live a consistently healthy lifestyle, month after month, year after year.

There is a problem with the production of these goods that is roughly analogous to the problem with the production of vague collective step goods. Consider, for instance, a person who enjoys the taste of junk food. He realizes that, strictly speaking, junk food is not good for his health, but like many of us, he thinks that the very small departure from his current level of physical functioning that, say, eating a candy bar causes can be outweighed by the sensory pleasure it provides. All things considered, it seems to him that trading off a miniscule decrease in his body's functioning in exchange for this sensory pleasure is better than not doing so. But, like many of us, he cannot imagine an amount of candy bar pleasure that it would be worth having if the price he must pay for it was to trade being healthy for being unhealthy. Presented with the options of being a healthy candy bar abstainer and an unhealthy candy bar glutton, he would say that the former would be the undeniably better life. By now, the problem these evaluative judgments raise should not be hard to spot. At any given moment, if he has the urge for a candy bar, he will judge that it is better to satisfy it than not, for the very slight decrease in his physical functioning seems to be worth the sensory satisfaction he stands to gain. And since it seems that there is no single candy bar that makes the difference between his being healthy and unhealthy, there is no point at which it seems to him that this judgment needs to be reversed. Because the issue is always whether to eat the candy bar and have his body function only minutely less well than it currently functions, and never whether to eat the candy bar and be unhealthy rather than healthy, he will continue to judge that it is better to satisfy his urges, only to wind up a positively unhealthy man. ${ }^{26}$

${ }^{26}$ This example preserves its force even when we assume that the man can detect the difference a single candy bar makes to his health: for it is still easy to imagine that this difference will be so slight that it is more than made up for by the pleasure of eating the candy bar. This makes the example different from, and less contentious than, that of the self-torturer presented in Quinn (1990). For Quinn's argument relies on the claim that undetected differences are not evaluatively 
Once again, it is difficult to explain where this man makes a mistaken evaluative judgment without having recourse to the notion that, with qualitative barrier cases, we can face tradeoffs involving borderline cases of evaluatively significant distinctions, and, as a result, can face tradeoffs regarding which we can neither affirm nor deny that they will yield a better result. Without this theoretical option, we would be committed to affirming of any given tradeoff that making it is either for the better, for the worse, or would yield no evaluative difference. And since it is very hard to see that the miniscule health effects of eating a single candy bar could really render doing so for the worse, we would then be committed to saying that the man makes a series of tradeoffs that are for the better, or at least that yield no evaluative difference, and yet is worse off for having done so than he would have been if he made none of the tradeoffs at all. ${ }^{27}$ The account offered here, however, allows us to say that when he is in the borderline region of being healthy, it would be a mistake to say that his trading off miniscule amounts of his physical health for sensory pleasure is always for the better. And this opens up the possibility of providing him with a reason to forgo that pleasure and, as a result, of saving him from making choices that will, eventually, render him unhealthy.

6. Qualitative barrier cases, then, constitute a theoretically challenging and practically important component of our evaluative lives. Once we understand their structure, we will also understand that we sometimes face decisions in which the best we can do is make a choice that is a borderline case of being for the better. And since our choices in such cases can lead us into real practical trouble especially if we misunderstand their evaluative status - there is a pressing need for a more widespread appreciation of the risks involved, and, ideally, for an account of our reasons for choosing that can rationally justify the series of decisions we need to make in order to avoid making things worse.

significant, and Arntzenius and McCarthy (1997) have given a compelling argument that that supposition is false.

27 Strictly speaking, it is possible that eating a single candy bar could be for the worse: if eating a single candy bar were to set off a cascade of biological effects, and these effects were sufficient to take one from a healthy state into an unhealthy one, then eating that candy bar would, in effect, involve trading off a healthy life for merely quantitative gain in pleasure. Note, however, that even when there is such a precise tipping point in the causal structure underlying a vague step good, we are typically deeply ignorant of where that tipping point lies. Consequently, we will be in an epistemic situation that is similar to the one we are in regarding vague step goods for which there is no underlying causal tipping point. For more on these points, see Knapp (forthcoming). 


\section{REFERENGES}

Arntzenius, Frank and McCarthy, David. (1997). "Self torture and group beneficence." Erkenntnis 47: 129-144.

Fine, Kit. (1975) "Vagueness, truth and logic." Synthese 30: 265-300.

Griffin, James. (1986) Well-Being: Its Meaning, Measurement, and Moral Importance. Oxford: Clarendon Press.

Hampton, Jean. (1987) "Free riders and collective goods." Economics and Philosophy 3: 245-273.

Kamm, Frances. (1996) Morality, Mortality, Volume II. Oxford: Oxford University Press.

Knapp, Christopher. (forthcoming) "Myopia, thresholds and precaution."

Machina, Kenton F. (1976) "Truth, belief and vagueness." Journal of Philosophical Logic 5: 47-78.

Norcross, Alastair. (1997) "Comparing harms: headaches and human lives." Philosophy and Public Affairs 26:135-167.

Parfit, Derek. (1987) "Overpopulation and the quality of life." in Peter Singer (ed.), Applied Ethics. Oxford: Oxford University Press.

Pettit, Philip. (1986) "Free riding and foul dealing." Journal of Philosophy 83: 361-379.

Quinn, Warren. (1990) "The puzzle of the self torturer." Philosophical Studies 59: 79-90.

Rachels, Stuart. (1998) "Counterexamples to the transitivity of better than." Australasian Journal of Philosophy 76: 71-83.

Raz, Joseph. (1986) The Morality of Freedom. Oxford: Clarendon Press.

Scanlon, T. M. (2001) What We Owe to Each Other. Cambridge: Harvard University Press.

Temkin, Larry, S. (2005) "A 'New' Principle of Aggregation," Philosophical Issues, 15: 218-234.

. (1996) "A continuum argument for intransitivity." Philosophy and

Public Affairs 25: 175-210. 
. (1987) "Intransitivity and the mere addition paradox." Philosophy and Public Affairs 16: 138-187.

Tuck, Richard (1987) "Is there a free-rider problem, and if so, what is it?" In Ross Harrison, ed., Rational Action. Cambridge: Cambridge University Press.

Williamson, Timothy. (1999) "On the Structure of Higher-Order Vagueness," Mind 108: 127-143.

. (1994) Vagueness. New York: Routledge. 